This item was submitted to Loughborough's Research Repository by the author.

Items in Figshare are protected by copyright, with all rights reserved, unless otherwise indicated.

\title{
Optimizing the secondary coil of a tesla transformer to improve spectral purity
}

PLEASE CITE THE PUBLISHED VERSION

http://dx.doi.org/10.1109/TPS.2013.2290763

PUBLISHER

(C) IEEE

VERSION

AM (Accepted Manuscript)

\section{PUBLISHER STATEMENT}

This work is made available according to the conditions of the Creative Commons Attribution-NonCommercialNoDerivatives 4.0 International (CC BY-NC-ND 4.0) licence. Full details of this licence are available at: https://creativecommons.org/licenses/by-nc-nd/4.0/

\section{LICENCE}

CC BY-NC-ND 4.0

\section{REPOSITORY RECORD}

Craven, Richard M., Ivor R. Smith, and Bucur M. Novac. 2019. "Optimizing the Secondary Coil of a Tesla Transformer to Improve Spectral Purity". figshare. https://hdl.handle.net/2134/20450. 


\title{
Optimising the secondary coil of a Tesla transformer to improve spectral purity
}

\author{
R M Craven, I R Smith and B M Novac
}

\begin{abstract}
This paper provides an overview of the response of the tuned secondary circuit of a Tesla transformer, following impulse excitation from the tuned primary circuit. Multi-order oscillatory voltages and currents are energised in the secondary circuit, and research is ongoing to determine the fundamental and higher-order modes for various secondary winding configurations, with the aim of developing design techniques that can be used to suppress the generation of the higher-order modes. It is anticipated that this will lead to generators which exhibit enhanced spectral purity and which will be better suited to use in electronic warfare applications than conventionally-wound Tesla transformers.
\end{abstract}

Keywords-filters, high-voltage techniques, pulsed power supplies, resonance, transformers

\section{INTRODUCTION}

$\mathbf{H}^{1}$ IGH voltage (HV) pulse generators capable of producing output pulses of more than $100 \mathrm{kV}$ in amplitude and with a very short rise time are a key requirement of many research activities, particularly in particle and plasma physics. Other examples of applications that directly utilise HV pulse generators include

- corona discharge for material processing

- x-ray generation

- high power laser research

- high power microwave (HPM) generation

HPM sources are currently under consideration as research tools for developing radar and electronic warfare (EW) capabilities. Illustrating the latter case as an example, one aspect of EW is that of intentional jamming, defined as [1] "the deliberate radiation ... of electromagnetic energy for the purpose of preventing or reducing an enemy's effective use of the electromagnetic spectrum". It is clear that the use of jamming techniques against an enemy must at the same time avoid disruption to own/allied radio communications (such as may be caused by unwanted harmonics from own/allied jamming sources for example). Careful spectrum management is required, namely the planning and coordination of the RF spectrum via diligent engineering, operational and administrative methodologies. In general and for any of the cases mentioned above, transferring technology from the laboratory to the field represents a significant engineering challenge regarding the pulsed power system that is required, and improvements are continually sought.

One familiar pulse generating source is the Tesla-based pulse transformer [2][3] that utilizes inductive coupling [4][5]

The authors are with the School of Electronic, Electrical and Systems Engineering, Loughborough University, Loughborough, Leicestershire, LE11 3TU, U.K. to transfer energy from a low impedance (primary) source to a higher impedance circuit and so produce an increase in the voltage generated across a load. The primary and secondary windings normally [6] have a coupling factor of 0.6 , and the two circuits (including the load impedance) are individually tuned to be resonant at the same frequency when decoupled from one another.

The typical circuit of Fig. 1 comprises a primary coil $L_{1}$, coupled via a mutual inductance $M$ to a secondary coil $L_{2}$, with the primary circuit tuned to be resonant by the external lumped capacitor $C_{1}$. The secondary circuit is similarly tuned to resonance by capacitance but this is usually the selfcapacitance of the secondary coil plus the capacitance of a field grading electrode [4] connected at the top of the secondary coil, plus its surroundings i.e. it is a distributed capacitance.

The secondary coil is usually grounded at the bottom end and the load and possibly a field grading electrode are affixed at the upper end, sometimes via a sharpening gap (a spark gap designed to hold off a high voltage before rapidly breaking down to apply a fast rising change in the voltage applied to the load). Maximum energy transfer to the load is obtained during the second half-cycle of the oscillatory response that follows the application of a voltage step to the primary circuit when the spark gap in the primary circuit is triggered.

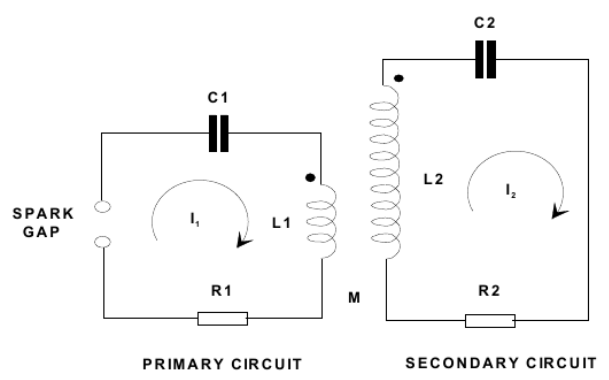

Figure 1. equivalent circuit of a Tesla transformer (sharpening gap and load not shown)

Quite separately, helical cavity filters [7] are high-Q devices similar to coaxial lines with helical inner conductors; they are used in radio frequency applications [8] such as filtering radio receiver inputs [9], or transmitter outputs of cellular phone base stations [10][11]. Although their application to high power pulse generation is not immediately obvious, a striking similarity exists between the geometry of a helical filter and the secondary coil of a Tesla transformer. This raises the possibility of utilising the techniques employed in the design of helical filters in Tesla transformer design, to examine any potential for enhanced performance. This prompted the 


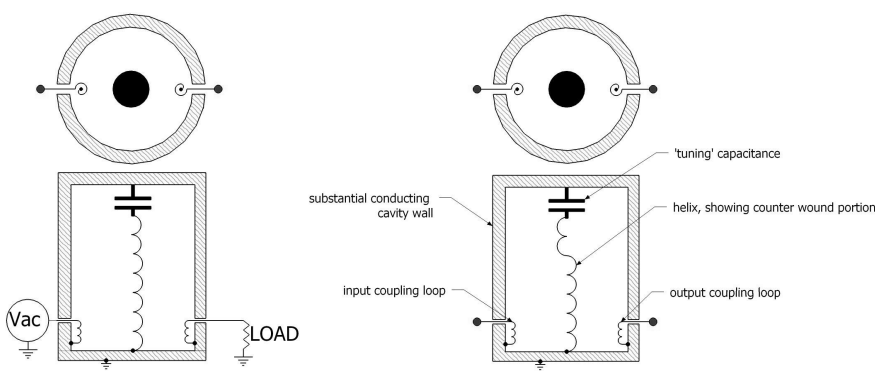

Figure 2. a typical coaxial cavity filter shown in-circuit, and illustrating reversed-pitch direction

investigation described below; it is hoped by the research to identify a new design of Tesla transformer secondary coil that generates a higher power output at the design frequency by diminution of its higher frequency content, thus also resulting in an improved spectral purity.

\section{HELICAL FILTERS}

In practice, a helical filter is typically constructed as a substantial, cylindrical, highly conductive cavity, often copper or silver plated, with a resonant structure along the central axis of the cavity and grounded at one end [12] as shown in Fig. 2. At lower frequencies (VHF and below) that inner resonant structure is often a helix in the form of a self-supporting aircored single-layer solenoid. An arrangement of loops provides coupling between the helix and the input and output signal.

The helix has an electrical length slightly less than a quarterwavelength of the resonant frequency, and is brought into resonance by the addition of the "top" capacitance, illustrated in Fig. 2 by the "T" shaped structures at the top of the helix. The helix and its distributed capacitance stores slightly less electric than magnetic field energy, with the shortfall made up by the top capacitor so that the overall arrangement is resonant. Several filters tuned to slightly different frequencies can be series connected to create a filter whose passband and stopband are tightly controlled.

It has been shown [13] that at its resonant frequency a helical filter (also termed a helical resonator) exhibits a minimum electric field strength at its grounded end and a maximum at the other end, whilst for the magnetic field it is a maximum at the grounded end and a minimum at the opposite end. Since the spatial separation between the two field maxima is a quarter wavelength $\left(\frac{\lambda}{4}\right)$ at the resonant frequency, a standing wave is set up along the resonant winding at both this wavelength and a series of related wavelengths when the electrical length of the helix is equivalent to [12]

$$
1 \frac{\lambda}{4}, 3 \frac{\lambda}{4}, 5 \frac{\lambda}{4}, \ldots \frac{(2 n+1) \lambda}{4}, n=1,2, \ldots
$$

which corresponds to a series of frequencies of

$$
f, 3 f, 5 f, \ldots(2 n+1) f, n=1,2, \ldots
$$

These standing wave terms represent a series of modes which describe the frequencies of currents flowing in the winding. In what follows the fundamental frequency is denoted by $f_{1}$, with $f_{3}$ being the frequency of the next mode (whose electrical length is $3 \frac{\lambda}{4}$ ) and so on.

This phenomenon illustrates a further parallel between a Tesla transformer secondary coil responding as a quarter-wave resonator and a cavity filter's shortened helix, since a Tesla transformer's secondary coil is brought into resonance by the additional capacitance of some form of electric field grading structure (such as a corona nut or metal toroid [4]) in just the same way as a helix in a cavity filter (see Fig. 2) is brought into resonance by additional capacitance at its high voltage end. It is thus further demonstrated that additional similarities exist between Tesla transformer secondary coils and helical filters with foreshortened ends. These similarities were illustrated by Sloan [14] who further developed the original Tesla transformer and, additionally, by Terman [15] who discussed the helical resonator in its cavity and evolved the description to that of an "open" helical resonator operating against a ground plane, with boundary conditions for the resonator removed. Vizmuller has demonstrated methods [13] by which standing waves can be suppressed on conducting helices (e.g. self-resonant coils) by changes in the helical pitch or diameter or in the direction of part of the winding.

A helix of fixed height $H=H_{1}+H_{2}$ can be constructed whereby $H_{1}$ is that portion of the helix wound in a normal direction whereas the remainder, $\mathrm{H}_{2}$, is counter-wound (i.e. the winding direction is reversed for portion $\mathrm{H}_{2}$ compared with that for portion $H_{1}$ ). It has been shown [13], and the present authors have confirmed by measurement, that the fundamental resonant frequency of the helix corresponds to $\frac{\lambda}{4}=H_{1}+H_{2}$, rather than $\frac{\lambda}{4}=H_{1}$ alone. However, the frequencies and magnitudes of the responses corresponding to the $f_{3}$ mode and above are particularly sensitive to the ratio of $H_{2}$ to $H_{1}$. Thus a mechanism exists whereby the higher-frequency mode responses can be significantly altered while the fundamental mode remains largely unaltered.

\section{Methodology}

A series of helical coils were constructed for experimental purposes. One was a conventional single- layer air-cored solenoid with 1600 turns, referred to as the "reference" or " $0 \%$ " coil because it had none of its turns counter wound, and the others were basically similar but with the top $10 \%, 22.5 \%$, $33 \%$ and $50 \%$ of the turns reversed. These sample dimensions were chosen in conjunction with ongoing computer modelling in order to help determine the optimum number of turns comprising the reversed winding direction, which maximises the increase in frequency for the higher-mode frequencies whilst leaving the $f_{1}$ mode frequency unaltered. In each case, identical acrylonitrile butadiene styrene (ABS) plastic formers were employed, constructed from $1 \mathrm{~mm}$ wall thickness tubes of $114 \mathrm{~mm}$ outer diameter and $570 \mathrm{~mm}$ length, upon which an identical length of enamelled copper wire was wound such that each coil was made to the same dimensions.

\section{A. $Q$ factor measurements}

A series of frequency domain measurements were made on the individual coils by energising them with a constant 
amplitude RF current, swept in frequency over a frequency range covering the fundamental resonant frequency and the first few higher modes. The fundamental resonant frequency and the $f_{3}, f_{5}$ and $f_{7}$ modes were calculated approximately from the self-inductance and self-capacitance of the coil and confirmed by measurement.

Reduction of quality factor at a particular mode frequency reduces the magnitude of the circulating current [15]. Analysis of the $Q$ factor of the experimental coils, at the various mode frequencies discussed, assists in determining the magnitudes of circulating currents at the various mode frequencies i.e. lowering of the $Q$ factor is coincident with lowering the magnitude of the current at that frequency (see Appendix). The quality factor of a resonant circuit, when energized by a current alternating at the circuit's resonant frequency, is defined as the ratio of energy lost per cycle to the energy stored per cycle [15]. At resonance, the complex input impedance is purely a real value; the imaginary components of the impedance cancel as the magnetic field energy and the electric field energy are equal. Above or below the resonant frequency the impedance appears complex; where the resistive part of the impedance is equal in magnitude to the complex part then

$$
R= \pm j X
$$

Equation 3 shows that the coil's effective inductive or capacitive reactance is equal in magnitude to the resistive part of the coil's impedance, and this occurs at a frequency either side of its resonant frequency. At these two frequencies, half of the power supplied to the resonator is dissipated in the resistive part and half is stored in a continuous exchange between the electric and magnetic fields which comprise the reactive part. If the complex impedance of the coil is measured by a vector network analyser (VNA) and displayed on a Smith chart, measuring this input impedance $(Z)$ at resonance and then finding the frequencies at which the inductive reactance and the capacitive reactance are equal in magnitude to one another enables the half-power points to be found for the fundamental and also higher mode resonances, denoted by $f_{n}$. The response can be measured since the lower $\left(f_{l}\right)$ and upper frequencies $\left(f_{u}\right)$, corresponding to a halving of input power, can be determined. Then $Q$ is given by [15]

$$
Q=\frac{f_{n}}{f_{u}-f_{l}}
$$

\section{B. In-circuit spectrum measurements}

A simple Tesla transformer with a secondary wound as a cylindrical solenoid was designed, constructed and used as a bench mark. The design goal was to achieve a repeatable high output voltage, employing simple components and air insulation at standard atmospheric pressure. Its secondary winding utilized a reference $(0 \%)$ coil, loaded by a field grading electrode [4] connected at the top of the secondary coil and with the resonant frequency of the Tesla transformer primary and secondary circuits tuned accordingly. A 200 $\mu A, 10 k V \mathrm{dc}$ to $\mathrm{dc}$ converter powered the transformer by slowly charging the primary capacitor until the spark gap fired.
A reliable and repeatable firing voltage was achieved every few seconds, enabling both single-shot measurements to be characterised and the secondary coil mode frequencies and currents to be compared with the previous $\mathrm{Q}$ measurements of the isolated secondary coil (see III-A). Numerous timedomain waveforms of the secondary current were captured using a Singer 91550-1 current transformer and a Tektronix digital oscilloscope, providing $100 \mathrm{MHz}$ of instrumentation bandwidth. Measurements were made using the $0 \%$ coil, then the other coils were substituted and the measurements were repeated. The data was numerically processed via a fast Fourier transform (FFT) to convolve a frequency domain spectrum. The resonant frequency and amplitude for the $f_{1}, f_{3}$ and $f_{5}$ modes were then observed for the $0 \%$ coil and compared with the $10 \%, 22.5 \%, 33 \%$ and $50 \%$ winding types.

Table I. EXPERIMENTAL TESLA TRANSFORMER PARAMETERS

\begin{tabular}{|l|l|l|}
\hline parameter & $\begin{array}{l}\text { calculated } \\
\text { value }\end{array}$ & $\begin{array}{l}\text { measured } \\
\text { value }\end{array}$ \\
\hline \hline Primary coil inductance $L_{P}$ & $70 \mu \mathrm{H}$ & $70 \mu \mathrm{H}$ \\
\hline Primary capacitance $C_{p}$ & $17.5 \mathrm{nF}$ & $17.5 \mathrm{nF}$ \\
\hline Primary capacitor stored energy & $875 \mathrm{~mJ}$ & $875 \mathrm{~mJ}$ \\
\hline Secondary coil inductance $L_{d c}$ & $45 \mathrm{mH}$ & $45 \mathrm{mH}$ \\
\hline Secondary coil capacitance $C_{s e c}$ & $10 \mathrm{pF}$ & $9.4 \mathrm{pF}$ \\
\hline Topload capacitance $C_{t o p}$ & $21 \mathrm{pF}$ & $20 \mathrm{pF}$ \\
\hline
\end{tabular}

\section{RESUlTS}

Measurements were made in a fixed location within a constant laboratory environment, comparing the reference coil to the re-wound versions, and the resonant frequencies and loaded $Q$ factors of the various mode responses are shown in tables II and III numerically and then in Fig. 3 graphically.

Operation in a Tesla transformer allowed spectra of the various coil types to be examined; for example the differing amplitude responses at the $f_{3}$ frequency are recorded in Table (IV).

Examination of the $Q$ factor data reveals some interesting details. It can be seen (Fig. 3 ) that the fundamental $\left(f_{1}\right)$ modes are grouped around $250 \mathrm{kHz}$ for all coil winding types with similar values for $Q$ of approximately 200 .

However, whilst the $f_{3}$ modes for all experimental coils show minor differences in mode frequency (all around $600 \mathrm{kHz}$ ), yet wider differences in the $f_{3}$ mode $Q$ factors are evident. Table II illustrates this numerically, showing that for the $f_{3}$ response in particular, an $11.2 \%$ mean decrease in $Q$ factor is observed (calculated from the bold values in table III) for the experimental coil types compared with the $0 \%$ reference coil.

Considering the $10 \%$ and $22.5 \%$ rewound coils, it can be seen that a decrease in $Q$ factor for both the $f_{3}$ and the $f_{5}$ modes is observed, whilst the fundamental shows a minor increase in the $Q$ factor. Comparison of this with the other rewound coils shows that for those other types, although demonstrating some specific reductions in $Q$ factor, their mean responses are not significantly different from the reference coil. 


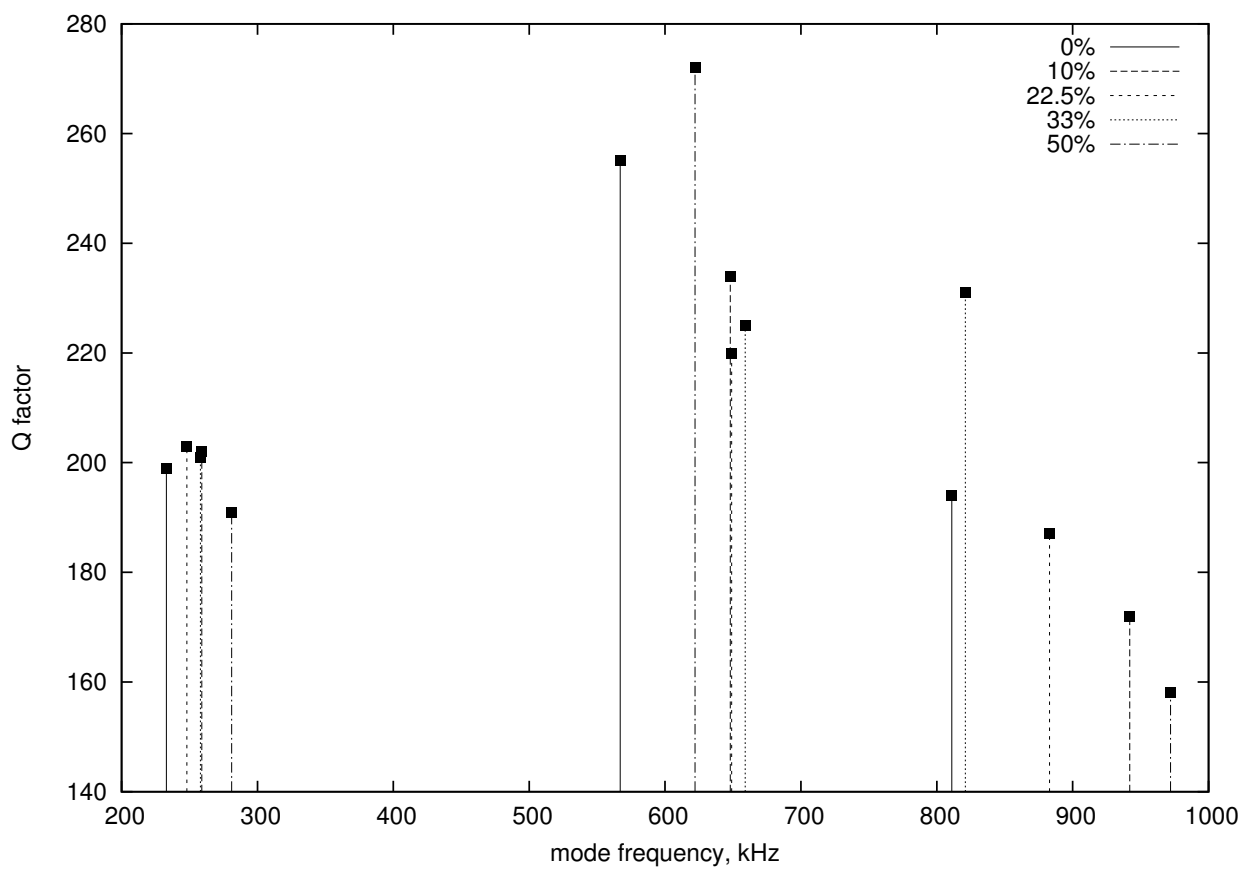

Figure 3. resonator mode frequencies and $Q$ factors: $f_{1}$ modes around $250 \mathrm{kHz}, f_{3}$ modes around $600 \mathrm{kHz}$ and $f_{5}$ modes above $800 \mathrm{kHz}$

Table II. MODE FREQUENCIES AND $Q$ FACTORS FOR REFERENCE AND REWOUND COILS

\begin{tabular}{|c|cc|cc|cc|cc|cc|}
\hline coil & \multicolumn{2}{|c|}{$0 \%$ (ref.) } & \multicolumn{2}{|c|}{$10 \%$} & \multicolumn{2}{c|}{$22.5 \%$} & \multicolumn{2}{c|}{$33 \%$} & \multicolumn{2}{c|}{$50 \%$} \\
\hline \hline mode & $\mathrm{kHz}$ & $Q_{L}$ & $\mathrm{kHz}$ & $Q_{L}$ & $\mathrm{kHz}$ & $Q_{L}$ & $\mathrm{kHz}$ & $Q_{L}$ & $\mathrm{kHz}$ & $Q_{L}$ \\
\hline$f_{1}$ & 233 & 199 & 259 & 202 & 248 & 203 & 258 & 201 & 281 & 191 \\
\hline$f_{3}$ & 567 & 255 & 648 & 234 & 649 & 220 & 659 & 225 & 622 & 272 \\
\hline$f_{5}$ & 811 & 194 & 942 & 172 & 883 & 187 & 821 & 231 & 972 & 158 \\
\hline
\end{tabular}

Table III. COIL RESPONSES AS \% CHANGES COMPARED WITH 0\% (REFERENCE) COIL, WITH SIGNIFICANT $Q$ FACTOR REDUCTIONS FOR THE $f_{3}$ MODE EMPHASIZED IN BOLD

\begin{tabular}{|c|c|c|c|c|c|c|c|c|c|c|}
\hline coil & \multicolumn{2}{|c|}{$0 \%$} & \multicolumn{2}{c|}{$10 \%$} & \multicolumn{2}{c|}{$22.5 \%$} & \multicolumn{2}{c|}{$33 \%$} & \multicolumn{2}{c|}{$50 \%$} \\
\hline mode & $\mathrm{kHz}$ & $Q_{L}$ & $\Delta k H z$ & $\Delta Q$ & $\Delta k H z$ & $\Delta Q$ & $\Delta k H z$ & $\Delta Q$ & $\Delta k H z$ & $\Delta Q$ \\
\hline$f_{1}$ & 233 & 199 & $11.2 \%$ & $1.5 \%$ & $6.4 \%$ & $2.0 \%$ & $10.7 \%$ & $1.1 \%$ & $20.6 \%$ & $-4.0 \%$ \\
\hline$f_{3}$ & 567 & 255 & $14.2 \%$ & $\mathbf{- 8 . 2 \%}$ & $14.5 \%$ & $\mathbf{- 1 3 . 7 \%}$ & $16.2 \%$ & $\mathbf{- 1 1 . 8 \%}$ & $9.7 \%$ & $6.7 \%$ \\
\hline$f_{5}$ & 811 & 194 & $16.2 \%$ & $-11.4 \%$ & $8.9 \%$ & $-3.6 \%$ & $1.2 \%$ & $19.0 \%$ & $19.9 \%$ & $-18.6 \%$ \\
\hline
\end{tabular}

In general for the modified coil windings, it can be seen that the higher-order modes experience a larger change in resonant frequency whilst the corresponding $Q$ factors diminish compared with the $0 \%$ (reference) coil. Studies of the mean values of the $Q$ factors and resonant frequencies of the various rewound coils show that, for the particular case of the $10 \%$ re-wound coil, its higher mode responses are highest in frequency and those associated higher modes have $Q$ factors which are lowest in value compared with the $0 \%$ reference coils. A $10 \%$ reduction in mean $Q$ factor is observed for the $10 \%$ coil whereas the $22.5 \%$ rewound coil has a mean $Q$ factor reduction of $8.7 \%$. The most significant decrease in $Q$ factor is observed in the $22.5 \%$ coil for its $f_{3}$ response.

In an investigation of the effects of introducing a counterwound section into a solenoid, Vizmuller [13] presented the reason why the fundamental mode frequency is unchanged. Counter winding of a section reduces the effective electrical length but introduces additional capacitance. This lowers the resonant frequency, but the electrical length of the solenoid $\mathrm{H}$ still remains a quarter of the wavelength at the fundamental mode frequency. Other authors (e.g. [7][8][12]) have demonstrated that when an RF current flows in a solenoid the effective electrical dimensions are a function of frequency. Thus, for example, the third harmonic becomes $3 f_{1}+\Delta f$. A detailed analysis of these modes is not trivial, but it seems likely that the counter winding process produces interference effects only at certain higher-order modes and not at the fundamental mode. 
Table IV. SUPPRESSION OF THE $f_{3}$ MODE's FREQUENCY RESPONSE IN A TESLA TRANSFORMER EMPLOYING THE VARIOUS SECONDARY COIL CONFIGURATIONS

\begin{tabular}{|c|c|c|c|c|c|}
\hline coil & $0 \%$ (ref.) & $10 \%$ & $22.5 \%$ & $33 \%$ & $50 \%$ \\
\hline \hline suppression $(\mathrm{dB})$ & 0 & $\mathbf{1 5 d B}$ & $0.8 \mathrm{~dB}$ & $1.6 \mathrm{~dB}$ & $0.2 \mathrm{~dB}$ \\
\hline
\end{tabular}

\section{CONCLUSIONS}

Design techniques used in helical cavity filters have been explored and applied to Tesla transformer secondary coils (i.e. single-layer solenoidal resonators). A measurement suite has been described which allows the fundamental and higher mode frequencies and currents of a range of experimental Tesla transformer secondary windings to be characterized. The authors have demonstrated that the magnitude of currents which flow at higher mode frequencies can be reduced whilst leaving the fundamental current amplitudes largely unchanged. The design techniques demonstrated may find utility in areas such as high power applications such as portable EMP generators [16] or as a pulsed power supply for driving loads such as experimental lasers [3] or radio frequency sources e.g. magnetrons for electronic warfare applications.

\section{APPENDIX}

At the high frequencies present in the secondary coil, the equivalent circuit takes the form shown in Fig. 4 where $R_{L O S S}$ takes into account the skin effect that will be present, with the $Q$ factor of the circuit defined by

$$
Q=\frac{\omega L}{R_{L O S S}}
$$

The voltage $V_{s}$ induced in the secondary winding by a current $I_{p}$ flowing in the primary circuit at frequency $\omega$ is

$$
V_{s}=-j \omega M I_{p}
$$

where $\mathrm{M}$ is the primary/secondary mutual inductance.

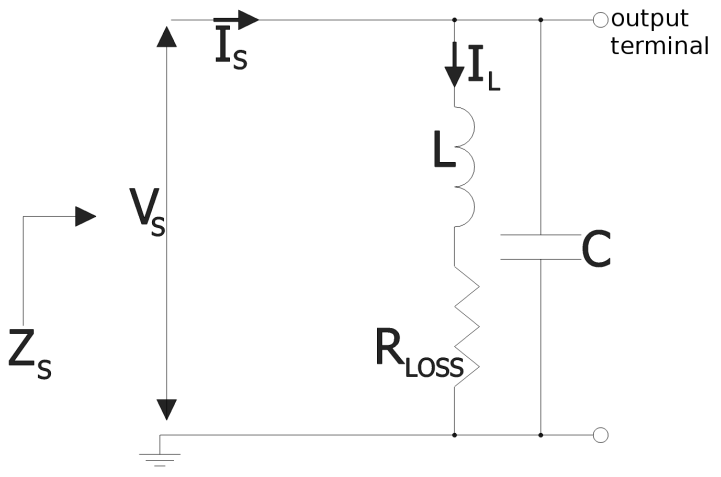

Figure 4. approximate equivalent circuit of unloaded secondary coil

It is straightforward to show that the circuit impedance at any resonant frequency shown by equation (2) is

$$
Z_{s}=R_{L O S S}\left(1+Q_{s}^{2}\right)
$$

where

$$
Q_{s}=\frac{1}{R_{L O S S}} \sqrt{\frac{L}{C}}
$$

Assuming the $Q$ factor of the coil is $>1$, then

$$
Z_{s} \cong R_{L O S S} Q_{s}^{2}=\frac{L}{C R_{L O S S}}
$$

and it is straightforward to show that the current $I_{L}$ flowing in the unloaded secondary circuit is

$$
I_{L}=I_{s} Q_{s}=\frac{I_{s}}{R_{L O S S}} \sqrt{\frac{L}{C}}
$$

The secondary current is thus directly proportional to $Q_{S}$ for any particular mode frequency. Thus, for example, for the $f_{3}$ mode and the $22.5 \%$ rewound experimental coil, a reduction in $Q$ factor of $13.7 \%$ (see table III) corresponds to a reduction of $13.7 \%$ in the current at that frequency, since

$$
\Delta I_{L}=I_{s} \Delta Q_{s}
$$

\section{REFERENCES}

[1] D. of the Army, Electronic Warfare in Operations (FM 3-36). US Army, available from https://armypubs.us.army.mil/doctrine/index.htm, 2012.

[2] G. N. Glasoe and J. V. Lebacqz, Pulse Generators. McGraw-Hill, 1948.

[3] W. J. Sarjeant and R. E. Dollinger, High Power Electronics. TAB Professional and Reference Books, 1989.

[4] J. Craggs and J. Meek, High Voltage Laboratory Technique. Butterworths Scientific Publications, 1954.

[5] R. Craven, "Design improvements in Tesla coil performance," in IEE Colloquium on Pulsed Power (Digest No: 1997/075), no. 75, 1997.

[6] M. Denicolai, "Optimal performance for tesla transformers," Review of Scientific Instruments, vol. 73, no. 9, pp. 3332-3336, 2002.

[7] A. Zverev, Handbook of Filter Synthesis. Wiley-Interscience, 1967.

[8] A. Kandoian and W. Sichak, "Wide-frequency-range tuned helical antennas and circuits," in IRE International Convention Record, vol. 1, pp. 42-47, 1953.

[9] J. Hong and M. Lancaster, Microstrip Filters for RF/microwave Applications. Wiley, 2001.

[10] P. Vizmuller, RF Design Guide, ch. Coupled Resonator Filters, pp. 114122. Artech House, 1995.

[11] Pasternack Enterprises, "GSM bandpass filter catalogue, $869 \mathrm{MHz}$ to 894 MHz. 2012 (3/3), pp. 1.” http://www.pasternack.com, 2012.

[12] W. W. Macalpine and R. O. Schildknecht, "Coaxial resonators with helical inner conductor," in Proc. IRE, pp. 2099-2105, 1959.

[13] P. Vizmuller, Filters with Helical and Folded Helical Resonators. Artech House, 1987

[14] D. H. Sloan, "A radiofrequency high-voltage generator," in Phys. Rev, vol. 47, 1935.

[15] F. Terman, Radio Engineer's Handbook. McGraw-Hill Handbooks, McGraw-Hill Book Company, Incorporated, 1943. 
[16] P. Sarkar, S. Braidwood, I. Smith, B. Novac, R. Miller, and R. Craven, "A compact battery-powered half-megavolt transformer system for EMP generation," Plasma Science, IEEE Transactions on, vol. 34, no. 5, pp. 1832-1837, 2006.

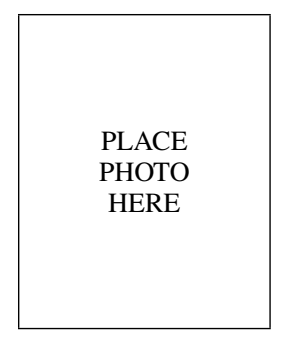

Richard M. Craven is studying part-time for a $\mathrm{Ph} . \mathrm{D}$ degree at Loughborough University; his career has spanned RF and microwave engineering (satcom development, design at system and component level and antenna design and development) and also aspects of pulsed power development, in particular Tesla transformers

Ivor. R. Smith received the B.Sc. and Ph.D. degrees from the University of Bristol, Bristol, U.K., after completing a student apprenticeship at the Witton Works, General Electric Company. He then became a Lecturer in electrical engineering with the University of Birmingham, Birmingham, U.K., subsequently being promoted to Lecturer and Reader and being awarded the degree of D.Sc. for his continued research contribution. He then moved to Loughborough University, Loughborough, U.K., to become a Professor of electrical power engineering, serving as the Head of Department, Dean of Engineering, and Pro-Vice Chancellor. For some 25 years, he has been active in research in many aspects of the production, conditioning, and utilization of large pulses of electrical energy, and his work has brought in very substantial funding from a wide range of sponsors. Dr. Smith is a Chartered Engineer. He is a Fellow of The Institution of Engineering and Technology and a Fellow of the Royal Academy of Engineering.

Bucur M. Novac (M'06 - SM'08) received the M.Sc. and Ph.D. degrees in 1977 and 1989, respectively. He joined the Loughborough University, UK in 1998 and is currently Professor of Pulsed Power. His research interests include compact high-power systems, explosively and electromagnetically driven magnetic flux compression generators and their applications, electromagnetic launchers, ultrafast magneto and electro-optic sensors and 2-D modeling of pulsed-power systems. Prof. Novac is a member of the International Steering Committees for both the MEGAGAUSS Conferences and for the Euro-Asian Pulsed Power Conferences. He is also member of the organizing committee for the IEEE International Power Modulator and High Voltage Conference and he also co-chairs the UK Pulsed Power Symposium. Prof. Novac is a Chartered Engineer and a Fellow of The Institution of Engineering and Technology. 